QUARTERLY OF APPLIED MATHEMATICS

VOLUME LXX, NUMBER 4

DECEMBER 2012, PAGES 665-683

S 0033-569X(2012)01257-3

Article electronically published on June 21, 2012

\title{
COUNTING THE SET OF EQUILIBRIA FOR A ONE-DIMENSIONAL FULL MODEL FOR PHASE TRANSITIONS WITH MICROSCOPIC MOVEMENTS
}

\author{
BY
}

JIE JIANG (Institute of Applied Physics and Computational Mathematics, PO Box 8009, Beijing 100088, People's Republic of China)

AND

YANYAN ZHANG (Department of Mathematics, East China Normal University, Shanghai 200241, People's Republic of China)

Abstract. This paper is concerned with the steady states of a one-dimensional strongly nonlinear PDE system arising from the study of phase transitions with microscopic movements. Using the plane analysis method, we prove that the number of equilibria is at most infinitely countable. Furthermore, we show that the global solution to the evolution system converges to an equilibrium in $H^{3} \times H^{1}$ as $t \rightarrow+\infty$.

1. Introduction. In this paper, we study the number of steady states and the convergence to equilibria for the following one-dimensional strongly nonlinear PDE system arising from the study of phase transitions with microscopic movements 5 :

$$
\begin{cases}f_{t}-\kappa f_{x x}+12 L f^{2}(f-1)+12 L \frac{\theta}{\theta_{c}} f(1-f)^{2}=0, & (x, t) \in(0, l) \times(0, \infty), \\ \alpha_{0} \theta_{t}-k_{0} \theta_{x x}-12 L \frac{\theta}{\theta_{c}} f(1-f)^{2} f_{t}-f_{t}^{2}=0, & (x, t) \in(0, l) \times(0, \infty), \\ \left.f_{x}\right|_{x=0, l}=0,\left.\quad \theta_{x}\right|_{x=0, l}=0, & t \in[0, \infty), \\ \left.f\right|_{t=0}=f_{0}(x),\left.\quad \theta\right|_{t=0}=\theta_{0}(x)>0, & x \in(0, l),\end{cases}
$$

where $\kappa, L, \theta_{c}, \alpha_{0}, k_{0}$ and $l$ are given positive constants. The state variables are the absolute temperature $\theta$ and the order parameter $f$ standing for the local proportion of one of the two phases.

Phase transition models with microscopic movements are developed based on Frémond's idea (see [3]) by having a recourse to a principle of virtual power and including the interior microscopic forces (cf., for instance, 1, 2, 5]). The resulting PDE system features a strongly nonlinear heat balance equation. Moreover, the total energy

Received October 23, 2010.

2000 Mathematics Subject Classification. Primary 34B08, 35B40; Secondary 35K45, 80A22.

Key words and phrases. Phase transitions, microscopic movements, steady states, convergence to equilibrium.

E-mail address: jiangbryan@gmail.com

E-mail address: yanyanzhangfd@yahoo.cn

(C) 2012 Brown University Reverts to public domain 28 years from publication 
of the system is conserved during the evolution process. Indeed, if we multiply the first equation of (1.1) by $f_{t}$, add the resultant to the second equation and integrate by parts, we obtain that

$$
\frac{d}{d t} \int_{0}^{l}\left(\alpha_{0} \theta+\frac{\kappa}{2} f_{x}^{2}+L\left(3 f^{4}-4 f^{3}\right)\right) d x=0 .
$$

Consequently, the following conservation relation holds:

$$
E(t):=\frac{1}{l} \int_{0}^{l}\left(\alpha_{0} \theta+\frac{\kappa}{2} f_{x}^{2}+L\left(3 f^{4}-4 f^{3}\right)\right) d x \equiv E(0):=m .
$$

Note that $m:=E(0)$ is determined by the initial data of problem (1.1). Then, it follows from the positivity of $\theta_{0}$ and $3 s^{4}-4 s^{3} \geq-1$ for $s \in \mathbb{R}$ that $m>-L$.

In view of (1.3), the corresponding stationary problem of system (1.1) reads:

$$
\left\{\begin{array}{l}
-\kappa v_{x x}+12 L v^{2}(v-1)+12 L \frac{u}{\theta_{c}} v(1-v)^{2}=0 \\
k_{0} u_{x x}=0, \\
\left.v_{x}\right|_{x=0, l}=0,\left.\quad u_{x}\right|_{x=0, l}=0 \\
\frac{1}{l} \int_{0}^{l}\left(\alpha_{0} u+\frac{\kappa}{2} v_{x}^{2}+L\left(3 v^{4}-4 v^{3}\right)\right) d x \equiv m .
\end{array}\right.
$$

Longtime behaviors of solutions to PDE systems for phase transition models based on Frémond's idea had been an open problem for years until Jiang and Guo [5] recently proved that the $\omega$-limit set $\omega\left(f_{0}, \theta_{0}\right)$ of system (1.1) is a nonempty connected compact set and consists of stationary solutions $(v, u)$ satisfying (1.4) and $u>0$ (see proof of Theorem 1.2). Consequently, in order to prove the convergence of any global solution to (1.1) towards equilibrium as time goes to infinity, we need to count the number of such solutions to (1.4) using the plane analysis method (see, e.g., [4, 20]).

By virtue of the homogeneous Neumann boundary conditions, we have

$$
u \equiv u^{0}>0,
$$

where $u^{0}$ is a positive constant that satisfies

$$
u^{0}=\frac{1}{\alpha_{0} l}\left[m l-\frac{\kappa}{2}\left\|v_{x}\right\|^{2}-L \int_{0}^{l}\left(3 v^{4}-4 v^{3}\right) d x\right] .
$$

It follows that the unknown function $v$ of (1.4) indeed satisfies a nonlinear elliptic equation with a nonlocal term (noticing (1.6))

$$
\left\{\begin{array}{l}
-\kappa v_{x x}+12 L v^{2}(v-1)+12 L \frac{u^{0}}{\theta_{c}} v(1-v)^{2}=0, \\
\left.v_{x}\right|_{x=0, l}=0
\end{array}\right.
$$

Note that multiplying the equation in (1.7) by $v$ and integrating on $(0, l)$ yields

$$
\kappa\left\|v_{x}\right\|^{2}+12 L \int_{0}^{l}\left(v^{4}-v^{3}\right) d x+12 L \frac{u^{0}}{\theta_{c}} \int_{0}^{l} v^{2}(1-v)^{2} d x=0 .
$$

Then a substitution of (1.8) into (1.6) leads to

$$
\alpha_{0} l u^{0}+L \int_{0}^{l}\left(2 v^{3}-3 v^{4}-\frac{6 u^{0}}{\theta_{c}} v^{2}(1-v)^{2}\right) d x=m l .
$$


By introducing a new parameter

$$
\sigma:=\frac{u^{0} / \theta_{c}}{1+u^{0} / \theta_{c}}
$$

and setting $a=\frac{\alpha_{0} \theta_{c}}{L}$, the stationary problem (1.4)-(1.5) can be rewritten as

$$
u \equiv u^{0}=\frac{\theta_{c} \sigma}{1-\sigma}>0
$$

and

$$
\left\{\begin{array}{l}
\frac{\kappa(1-\sigma)}{L} v_{x x}=P(v ; \sigma) \triangleq 12 v(1-v)(\sigma-v), \\
\left.v_{x}\right|_{x=0, l}=0, \\
\frac{1}{l} \int_{0}^{l}\left[\frac{a \sigma}{1-\sigma}+2 v^{3}-3 v^{4}-\frac{6 \sigma}{1-\sigma} v^{2}(1-v)^{2}\right] d x \equiv m / L .
\end{array}\right.
$$

In this paper, we show that problem (1.11)-(1.12) admits at most an infinitely countable number of regular solutions $\left(v(x), u^{0}\right)$. Then applying the well-known results for gradient systems (see [25, 26]), we conclude that any global solution to the evolution equation (1.1) will converge to a single stationary solution to (1.11)-(1.12) as time tends to infinity.

More precisely, the main results of this paper are the following theorems:

Theorem 1.1. For any given positive constants $\kappa, L, \alpha_{0}, \theta_{c}, l$ and $m$ determined by (1.3), problem (1.11)-(1.12) has at most an infinitely countable number of solutions. More precisely, we have

(1) For $l \leq \underline{\lambda}:=\pi \sqrt{\frac{k}{12 L}}$, problem (1.11)-(1.12) only has trivial solutions.

(2) For $l>\underline{\lambda}$, problem (1.11)-(1.12) has a finite number of solutions in the following cases:

(a) $a_{1}<a$ and $m / L \in(-1,0]$, where $a_{1}=12 \sigma^{* 2}\left(1-\sigma^{*}\right)^{3}$ and $\sigma^{*}=\frac{21-\sqrt{57}}{24}$;

(b) $0<a \leq a_{1}$ and $m / L \in\left(-1, g\left(\sigma_{2}\right)\right)$, where $g(\sigma)=3 \sigma^{4}-4 \sigma^{3}+\frac{a \sigma}{1-\sigma}$ and $\sigma_{2}$ is the minimum value point of $g(\sigma)$ on $(0,1)$;

(c) $a, m / L$ are not in the cases $(a)$ or $(b)$, and $m / L \neq g\left(\sigma_{0}\right)$ for any $n \in$ $\{1,2, \ldots,[l / \underline{\lambda}]\}$, where $\sigma_{0}=\frac{\pi^{2} k}{12 L}\left(\frac{n}{l}\right)^{2}$.

(3) Otherwise, problem (1.11)-11.12) has at most an infinitely countable number of solutions.

Theorem 1.2. For any $\left(f_{0}, \theta_{0}\right) \in H^{3} \times H^{1}$ satisfying $\left.f_{0 x}\right|_{x=0, l}=0$ and $\theta_{0}>0$ in $[0, l]$, the unique global-in-time solution $(f, \theta)$ of problem (1.1) converges to an equilibrium in $H^{3} \times H^{1}$ as $t \rightarrow+\infty$.

Before giving the detailed proof of our main results, we first recall some related results in the literature.

In recent years, PDE systems for phase transition models taking microscopic movements into account have been extensively studied in many research papers. It was first proposed by Bonfanti, Frémond and Luterotti in [2] (see, also [3]) that the power of interior forces depends on microscopic movements. From a mathematical point of view, the resulting PDE system is characterized by a strongly nonlinear heat balance equation and the total energy should obey a conservation relation in analogy to (1.3). As far as we know, global well-posedness results for such PDE systems were only obtained in the 
one-dimensional case; see [6, 10, 11, 17] and the references cited therein. However, due to the difficulties brought by the strong nonlinearities of the original full models, longtime behaviors of the global solutions were only studied for simplified models by neglecting the highly nonlinear terms (see, for instance, [17]). It is worth noting that in [5] the authors first investigated the longtime behavior of the global solutions to a full phase transition model with microscopic movements. More precisely, by establishing a lemma in analysis they obtained the uniform boundedness of the global solutions to system (1.1). Moreover, compactness of the orbit and existence of global attractors were obtained as well. However, convergence to equilibrium of any single trajectory was not considered in that paper. The present paper is a continuation of [5].

Convergence of global solutions to equilibria for nonlinear evolution equations has been a problem of great interest and importance for a long time. For the one-dimensional case, significant progress has been made, as can be seen from the work by H. Matano [12] in 1978 and other works later on. For the higher space dimension case, it has been a focus of many researchers since the work by L. Simon 21] that extended a lemma by S. Lojasiewicz on analytic functions (cf. [7, 8, 9]) to the infinite-dimensional case and developed a method for the study of convergence to the stationary solution when the nonlinear term is analytic. We refer the readers to [26, Chapter 6] and the references cited therein for detailed discussions for the higher-dimensional case.

For the one space dimension case, once we prove that the set of equilibria is discrete, the convergence results immediately follow from the connectedness of the $\omega$-limit set consisting of the stationary solutions. As far as counting the number of steady-state solutions in the one space dimensional case is concerned, we study the number of solutions to problem (1.11)-(1.12) roughly following the idea proposed by Zheng and Novick-Cohen (see, e.g., 4, 14, 15, 24). The basic idea of the method is to convert the problem to finding intersection points of two analytic functions of one variable. Several equations have been treated in the literature. For instance, the Sivashinsky equation in [13, 15, the Cahn-Hilliard equation in [4, 14, 24, the Penrose-Fife-type equations in [16], the coupled Cahn-Hilliard equations in [20, 26], the thin-film equation in 22] and the chemotaxis model in 23], etc.

Before concluding this part, we want to stress some new features of the present paper.

(I) We note that the conservation identity as well as the nonlinear elliptic equation for $v$ with nonlocal term are difficult to handle since a derivative term $v_{x}^{2}$ is involved. In order to cancel the derivative term we use the energy method which leads to a fourth-order polynomial in the integral (1.12) (c) instead. Nevertheless, the correspondingly defined $R(\sigma, b)$ appearing in Section 2 is rather complicated to be dealt with.

(II) Due to the complexity of $I_{0}$ and $R$ defined in Section 2, it is no longer easy for us to obtain the monotonicity of them by examining the integration itself as is done in the literature. In fact, we follow a modified approach proposed by Zhang [22, 23] to study the properties of $I_{0}$ and $R$. However, we need to point out that in our problem the sketches of the level sets $\mathscr{C}_{\lambda}$ and $\mathscr{D}_{\mu}$ defined in Section 5 are quite different from those of [22, 23]. We need more careful discussions on the properties of $\mathscr{C}_{\lambda}$ and $\mathscr{D}_{\mu}$ (see Proposition 5.2 and Corollary 5.1). 
(III) Noticing that the absolute temperature $u^{0}$ can be arbitrarily large, we need to introduce a new parameter $\sigma$ defined by (1.10). This guarantees the boundedness of the admissible region.

This paper is organized as follows. In Section 2, we show some properties of the solutions to problem (1.11)-(1.12) and we introduce the admissible region for problem (1.11)-(1.12). In Section 3, we obtain the asymptotic behavior of the functions $I_{0}(\sigma, b)$ and $R(\sigma, b)$ when $(\sigma, b) \in \Sigma$ approaches the boundary of $\Sigma$. In Section 4 , we examine the monotonicity of $I_{0}$ with respect to $b$. In Section 5 , we analyze the level sets of the functions $I_{0}$ and $R$. At last, exploiting the analyticity of the functions $I_{0}$ and $R$, we conclude the main results, Theorem 1.1 and 1.2 in Section 6 .

2. The admissible region. In this section, we introduce the admissible region for problem (1.11)-(1.12). Since $u^{0}>0$, we have $0<\sigma<1$ by (1.11). To begin with, we list the following properties of solutions to problem (1.12) without proof (see [23]):

(P1) There exist at most five trivial solutions $\left(v, u^{0}\right)$ to problem (1.11)-(1.12) (see Figure 3 and Proposition 5.2).

(P2) Each nontrivial solution $v$ to problem (1.12) is periodic. More precisely, it consists of finite pieces of monotone branches, and it is symmetric with respect to any critical point of $v(x)$. In other words, if $v_{x}(\bar{x})=0, \bar{x} \in(0, l)$, then $v(\bar{x}-b)=v(\bar{x}+b)$ for any $b \in \mathbb{R}$ satisfying that both $\bar{x}-b$ and $\bar{x}+b$ belong to $[0, l]$.

(P3) Suppose $v$ is a nontrivial solution to problem (1.12), and $0, x_{1}$ are the two smaller critical points of $v(x)$. Then there exists another solution $\tilde{v}$ (called the reflection of $v$ ), which satisfies $\tilde{v}(x)=v\left(x_{1}-x\right)$ for $x \in\left[0, x_{1}\right]$. It is obvious that $v$ and $\tilde{v}$ have the same period and opposite monotonic property.

(P4) By the maximum principle, each solution $v$ satisfies $0 \leq v \leq 1$.

Now, multiplying (1.12) (a) by $v_{x}$, we obtain the following energy identity:

$$
\frac{\kappa(1-\sigma)}{2 L} v_{x}^{2}=F(v ; \sigma, b):=\Phi(v ; \sigma)-b
$$

where

$$
\Phi(v ; \sigma)=3 v^{4}-4(1+\sigma) v^{3}+6 \sigma v^{2}
$$

and $b$ is some constant of integration.

In view of (2.1), if $v$ is a nontrivial solution, there exists a pair of parameters $(\sigma, b)$ such that $F$ has at least two different zeros $v_{1}$ and $v_{2}$, so that

$$
\Phi(v(x) ; \sigma)-b>0 \text { for } v_{1}<v<v_{2} .
$$

Such a pair $(\sigma, b)$ will be called admissible and the union of all admissible pairs will be called the admissible region and will be denoted by $\Sigma$, i.e.,

$$
\Sigma=\left\{(\sigma, b) \mid \begin{array}{r}
\sigma \in(0,1), \exists v_{1}(\sigma, b)<v_{2}(\sigma, b), \text { s.t. } F\left(v_{i}\right)=0(i=1,2) \\
\text { and } F(v)>0, \text { when } v \in\left(v_{1}, v_{2}\right)
\end{array}\right\} .
$$

From (2.1) we also infer that

$$
d x= \pm \sqrt{\frac{\kappa(1-\sigma)}{2 L}} \frac{d v}{\sqrt{F(v ; \sigma, b)}} .
$$


Suppose $v$ is a simple solution on $\left[0, I_{0}\right]$, i.e., $v$ is monotone on $\left[0, I_{0}\right]$ and $v_{x}(0)=v_{x}\left(I_{0}\right)=$ 0 . Without loss of generality, we assume that $v^{\prime}>0$ on $\left(0, I_{0}\right)$. Then $v(0)=v_{1}, v\left(I_{0}\right)=v_{2}$. From (2.5), we get

$$
I_{0}(\sigma, b)=\sqrt{\frac{\kappa(1-\sigma)}{2 L}} \int_{v_{1}}^{v_{2}} \frac{d s}{\sqrt{F(s ; \sigma, b)}},
$$

which, as in Schaaf [18, 19, is called the time map. Moreover, by (P2) there exists $n \in \mathbb{N}$ such that

$$
I_{0}(\sigma, b)=l / n \text {. }
$$

We also define

$$
\begin{aligned}
I_{1}(\sigma, b) & =\sqrt{\frac{\kappa(1-\sigma)}{2 L}} \int_{v_{1}}^{v_{2}}\left\{2 s^{3}-3 s^{4}-\frac{6 \sigma}{1-\sigma} s^{2}(1-s)^{2}+\frac{a \sigma}{1-\sigma}\right\} \frac{d s}{\sqrt{F(s ; \sigma, b)}}, \\
R(\sigma, b) & =\frac{I_{1}(\sigma, b)}{I_{0}(\sigma, b)} .
\end{aligned}
$$

Now our problem is reduced to finding $(\sigma, b) \in \Sigma$ such that (2.7) holds for some $n \in \mathbb{N}$ and $R(\sigma, b)=m / L$.

In the rest of this section, we study the sketch of the admissible region. Let us begin with the investigation of $P(v ; \sigma)$ and $\Phi(v ; \sigma)$.

Suppose

$$
P(w ; \sigma)=0
$$

Then we have

$$
w_{0}=0, \quad w_{1}=\sigma, \quad w_{2}=1,
$$

and when $\sigma \in(0,1)$,

$$
P_{v}\left(w_{i} ; \sigma\right)>0, \quad \text { for } i=0,2 ; \quad P_{v}\left(w_{1} ; \sigma\right)<0 .
$$

It can be seen that the equation $\Phi(v ; \sigma)-b=0$ has four roots $v_{i}(i=0,1,2,3)$ satisfying $v_{0} \leq w_{0} \leq v_{1} \leq w_{1} \leq v_{2} \leq w_{2} \leq v_{3}$ and (2.3) if and only if $\sigma \in(0,1)$, $b \in[\Phi(0), \Phi(\sigma))$ for $\sigma \in\left(0, \frac{1}{2}\right]$ and $b \in[\Phi(1), \Phi(\sigma))$ for $\sigma \in\left[\frac{1}{2}, 1\right)$. A sketch of $\Phi(v ; \sigma)$ when $0<\sigma<\frac{1}{2}$ is shown in Figure 1

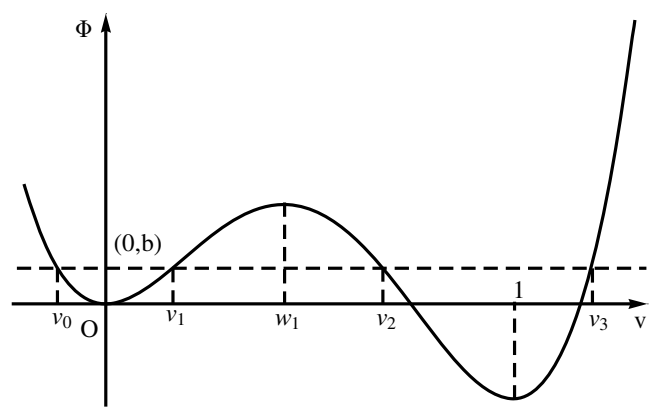

FIG. 1. A sketch of $\Phi(v ; \sigma)$ when $0<\sigma<\frac{1}{2}$. 
On the basis of the above analysis, the admissible region $\Sigma$ can be expressed as

$$
\Sigma=\left\{(\sigma, b) \mid \begin{array}{r}
\sigma \in(0,1), \Phi(0) \leq b<\Phi(\sigma) \text { for } \sigma \in\left(0, \frac{1}{2}\right] \\
\text { and } \Phi(1) \leq b<\Phi(\sigma) \text { for } \sigma \in\left[\frac{1}{2}, 1\right)
\end{array}\right\} .
$$

Furthermore, define

$$
\begin{aligned}
& \Gamma_{0}: b=b_{0}(\sigma)=\Phi\left(w_{0} ; \sigma\right)=0, \quad 0<\sigma \leq \frac{1}{2}, \\
& \Gamma_{1}: b=b_{1}(\sigma)=\Phi\left(w_{1} ; \sigma\right)=\sigma^{3}(2-\sigma), \quad 0<\sigma<1, \\
& \Gamma_{2}: b=b_{2}(\sigma)=\Phi\left(w_{2} ; \sigma\right)=2 \sigma-1, \quad \frac{1}{2} \leq \sigma<1, \\
& O=(0,0), \quad A=\left(\frac{1}{2}, 0\right), \quad B=(1,1) .
\end{aligned}
$$

We remark that $\Gamma_{0}$ and $\Gamma_{2}$ belong to $\Sigma$ while $\Gamma_{1}$ does not. A sketch of $\Sigma$ is shown in Figure 2,

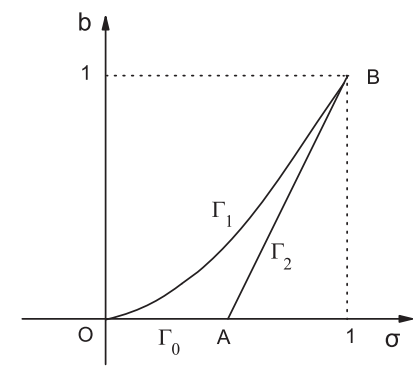

FIG. 2. A sketch of admissible region $\Sigma$.

3. Properties of $I_{0}(\sigma, b)$ and $R(\sigma, b)$ along $\partial \Sigma$. In this section, we study the properties of $I_{0}(\sigma, b)$ and $R(\sigma, b)$ along $\Gamma_{0}, \Gamma_{1}$ and $\Gamma_{2}$. First, we give the following convenience expressions for $I_{0}$ and $I_{1}$ (see [14]).

Lemma 3.1. For $(\sigma, b) \in \Sigma$, we have that

$$
\begin{aligned}
& \sqrt{\frac{2 L}{k(1-\sigma)}} I_{0}(\sigma, b)=\sum_{i=1}^{2} \int_{0}^{1} \frac{d x}{\sqrt{(1-x) Q\left(x ; v_{i}, w_{1}\right)}}, \\
& \sqrt{\frac{2 L}{k(1-\sigma)}} R(\sigma, b)=\sum_{i=1}^{2} \int_{0}^{1} \frac{H\left(x ; v_{i}, w_{1}\right) d x}{\sqrt{(1-x) Q\left(x ; v_{i}, w_{1}\right)}},
\end{aligned}
$$

where

$$
\begin{aligned}
Q\left(x ; v, w_{1}\right)= & -3 v^{2}\left(x^{3}+x^{2}+x+1\right)+v w_{1}\left(6 x^{3}-2 x^{2}-2 x-2\right) \\
& -w_{1}^{2}\left(3 x^{3}-5 x^{2}+x+1\right)+4 v\left(x^{2}+x+1\right)-2 w_{1}\left(2 x^{2}-x-1\right)
\end{aligned}
$$


and

$$
\begin{aligned}
H\left(x ; v, w_{1}\right)=\frac{a w_{1}}{1-w_{1}} & +2\left(\left(v-w_{1}\right) x+w_{1}\right)^{3}-3\left(\left(v-w_{1}\right) x+w_{1}\right)^{4} \\
& -\frac{6 w_{1}}{1-w_{1}}\left(\left(v-w_{1}\right) x+w_{1}\right)^{2}\left(1-\left(\left(v-w_{1}\right) x+w_{1}\right)\right)^{2} .
\end{aligned}
$$

Proof. Defining a new variable $t=s-w_{1}$ and splitting the range of integration into two parts, we deduce that

$$
\begin{aligned}
\sqrt{\frac{2 L}{k(1-\sigma)}} I_{0}(\sigma, b) & =\int_{v_{1}}^{v_{2}}\left(3 s^{4}-(4+4 \sigma) s^{3}+6 \sigma s^{2}-b\right)^{-\frac{1}{2}} d s \\
& =\int_{v_{1}-w_{1}}^{v_{2}-w_{1}}\left[3\left(t+w_{1}\right)^{4}-(4+4 \sigma)\left(t+w_{1}\right)^{3}+6 \sigma\left(t+w_{1}\right)^{2}-b\right]^{-\frac{1}{2}} d t \\
& =-J_{1}+J_{2},
\end{aligned}
$$

where

$$
J_{i}=\int_{0}^{v_{i}-w_{1}}\left[3\left(t+w_{1}\right)^{4}-(4+4 \sigma)\left(t+w_{1}\right)^{3}+6 \sigma\left(t+w_{1}\right)^{2}-b\right]^{-\frac{1}{2}} d t
$$

for $i=1,2$.

Now introducing the variable $x_{i}=t /\left(v_{i}-w_{1}\right)$ in the integral $J_{i}$ and noticing that $b=\Phi\left(v_{i}\right)$, we obtain that (omitting the subscript $i$ )

$$
\begin{gathered}
J=\int_{0}^{v-w_{1}}\left[3\left(t+w_{1}\right)^{4}-(4+4 \sigma)\left(t+w_{1}\right)^{3}+6 \sigma\left(t+w_{1}\right)^{2}\right. \\
\left.-\left(3 v^{4}-(4+4 \sigma) v^{3}+6 \sigma v^{2}\right)\right]^{-\frac{1}{2}} d t \\
=\int_{0}^{1}\left\{3\left[\left(x\left(v-w_{1}\right)+w_{1}\right)^{4}-v^{4}\right]-(4+4 \sigma)\left[\left(x\left(v-w_{1}\right)+w_{1}\right)^{3}-v^{3}\right]\right. \\
\left.\quad+6 \sigma\left[\left(x\left(v-w_{1}\right)+w_{1}\right)^{2}-v^{2}\right]\right\}^{-\frac{1}{2}}\left(v-w_{1}\right) d x \\
=\int_{0}^{1}\left[\left(v-w_{1}\right)^{2}(1-x) Q\left(x ; v, w_{1}\right)\right]^{-\frac{1}{2}}(v-w) d x .
\end{gathered}
$$

Then (3.1) follows. The expression for $I_{1}(\sigma, b)$ is obtained following the same procedure. This completes the proof.

Now, based on Lemma 3.1, we discuss the behavior of $I_{0}$ and $R$ as we approach $\Gamma_{1}$ along lines of constant $\sigma$. 
Proposition 3.1. Let $\sigma \in(0,1)$ be fixed. Then it follows that as $b \uparrow b_{1}(\sigma)$,

$$
\begin{aligned}
I_{0}(\sigma, b) \rightarrow & \sqrt{\frac{k(1-\sigma)}{L}} \frac{\pi}{\sqrt{-P_{v}\left(w_{1} ; \sigma\right)}}=\sqrt{\frac{k}{L}} \frac{\pi}{\sqrt{12 \sigma}}, \\
I_{1}(\sigma, b) \rightarrow & \sqrt{\frac{k(1-\sigma)}{L}} \frac{\pi}{\sqrt{-P_{v}\left(w_{1} ; \sigma\right)}}\left[2 w_{1}^{3}-3 w_{1}^{4}-\frac{6 \sigma}{1-\sigma} w_{1}^{2}\left(1-w_{1}\right)^{2}+\frac{a \sigma}{1-\sigma}\right] \\
& =\sqrt{\frac{k}{L}} \frac{\pi}{\sqrt{12 \sigma}}\left(3 \sigma^{4}-4 \sigma^{3}+\frac{a \sigma}{1-\sigma}\right) \\
R(\sigma, b) \rightarrow & 3 \sigma^{4}-4 \sigma^{3}+\frac{a \sigma}{1-\sigma} .
\end{aligned}
$$

Proof. Now define $b=b_{1}(\sigma)+\frac{1}{2} P_{v}\left(w_{1}\right) \varepsilon^{2}$. Then it is straightforward to check that $v_{1}$ and $v_{2}$ satisfy

$$
v_{1}=w_{1}-\varepsilon+O\left(\varepsilon^{2}\right) \text { and } v_{2}=w_{1}+\varepsilon+O\left(\varepsilon^{2}\right),
$$

and hence for $i=1,2$, it follows that

$$
Q\left(x ; v_{i}, w_{1}\right)=6\left(w_{1}-w_{1}^{2}\right)(1+x)+O(\varepsilon) .
$$

Substituting this expression in (3.1), the asymptotic limit of $I_{0}$ is obtained. The asymptotic limit of $I_{1}$ can be obtained analogously and then the asymptotic limit of $R$ follows.

The behavior of $I_{0}(\sigma, b)$ and $R(\sigma, b)$ as we approach $\Gamma_{0}$ along lines of constant $\sigma$ is given in the following proposition.

Proposition 3.2. Let $\sigma \in\left(0, \frac{1}{2}\right)$ be fixed. Then it follows that as $b \downarrow b_{0}(\sigma)$,

$$
\begin{aligned}
& I_{0}(\sigma, b) \rightarrow \frac{1}{2} \sqrt{\frac{k(1-\sigma)}{L}} \frac{1}{\sqrt{P_{v}\left(w_{0} ; \sigma\right)}} \log \frac{1}{b-b_{0}(\sigma)}+O(1), \\
& I_{1}(\sigma, b) \rightarrow \frac{1}{2} \sqrt{\frac{k(1-\sigma)}{L}} \frac{\log \frac{1}{b-b_{0}(\sigma)}}{\sqrt{P_{v}\left(w_{0} ; \sigma\right)}}\left[2 w_{0}^{3}-3 w_{0}^{4}-\frac{6 \sigma w_{0}^{2}\left(1-w_{0}\right)^{2}}{1-\sigma}+\frac{a \sigma}{1-\sigma}\right]+O(1), \\
& R(\sigma, b) \rightarrow \frac{a \sigma}{1-\sigma} .
\end{aligned}
$$

Proof. Define $b=\frac{1}{2} P_{v}\left(w_{0}\right) \varepsilon^{2}=6 \sigma \varepsilon^{2}$. Then it is staightforward to check that $v_{1}$ and $v_{2}$ satisfy

$$
v_{1}=\varepsilon+O\left(\varepsilon^{2}\right)=\varepsilon s_{1}(\varepsilon) \quad \text { and } \quad v_{2}=\tilde{v}-O\left(\varepsilon^{2}\right)=\varepsilon s_{2}(\varepsilon),
$$

where $\tilde{v}$ is the solution to $\Phi(v)=0$ and satisfies $w_{1}<\tilde{v}<w_{2}=1, s_{1}=1+O(\varepsilon)$ and $s_{2}=\frac{\tilde{v}-O\left(\varepsilon^{2}\right)}{\varepsilon}$. Defining a new variable $s=v / \varepsilon$ and splitting the range of integration into 
two parts, we arrive at

$$
\begin{aligned}
\int_{v_{1}}^{v_{2}} \frac{d v}{\sqrt{F(v ; \sigma, b)}} & =\int_{\varepsilon+O\left(\varepsilon^{2}\right)}^{\tilde{v}-O\left(\varepsilon^{2}\right)} \frac{d v}{\sqrt{F(v ; \sigma, b)}} \\
& =\int_{s_{1}}^{s_{2}} \frac{\varepsilon d s}{\sqrt{F(\varepsilon s ; \sigma, b)}} \\
& =\int_{s_{1}}^{\frac{s_{1}+s_{2}}{2}} \frac{\varepsilon d s}{\sqrt{F(\varepsilon s ; \sigma, b)}}+\int_{\frac{s_{1}+s_{2}}{2}}^{s_{2}} \frac{\varepsilon d s}{\sqrt{F(\varepsilon s ; \sigma, b)}} \\
& \triangleq A_{1}+A_{2},
\end{aligned}
$$

where $A_{1}$ can be written as

$$
\begin{aligned}
A_{1} & =\int_{s_{1}}^{\frac{s_{1}+s_{2}}{2}}\left(\frac{\varepsilon}{\sqrt{F(\varepsilon s ; \sigma, b)}}-\frac{1}{\sqrt{\frac{P_{v}\left(w_{0}\right)}{2}}} \frac{1}{s}\right) d s+\int_{s_{1}}^{\frac{s_{1}+s_{2}}{2}} \frac{1}{\sqrt{\frac{P_{v}\left(w_{0}\right)}{2}}} \frac{1}{s} d s \\
& \triangleq A_{11}+A_{12} .
\end{aligned}
$$

Since

$$
\ln \frac{s_{1}+s_{2}}{2 s_{1}}-\ln \frac{1}{\varepsilon} \rightarrow \ln \frac{\tilde{v}}{2}, \quad \text { as } \quad \varepsilon \rightarrow 0
$$

we have that

$$
A_{12}=\frac{1}{\sqrt{\frac{P_{v}\left(w_{0}\right)}{2}}} \ln \frac{1}{\varepsilon}+O(1), \quad \text { as } \quad \varepsilon \rightarrow 0 .
$$

Now introducing a new variable $z=\left(s-\frac{s_{1}+s_{2}}{2}\right) /\left(\frac{s_{1}-s_{2}}{2}\right)$, we get

$$
A_{11}=\int_{0}^{1}\left(\frac{\varepsilon \frac{s_{2}-s_{1}}{2}}{\sqrt{F\left(\varepsilon\left(\frac{s_{1}-s_{2}}{2} z+\frac{s_{1}+s_{2}}{2}\right) ; \sigma, b\right)}}-\frac{1}{\sqrt{\frac{P_{v}\left(w_{0}\right)}{2}}} \frac{1}{\frac{s_{1}+s_{2}}{s_{2}-s_{1}}-z}\right) d z
$$

Denoting

$$
c=\tilde{v} / 2,
$$

recalling the definition of $s_{1}$ and $s_{2}$, by calculating we infer that

$$
\begin{gathered}
\varepsilon\left(\frac{s_{1}-s_{2}}{2} z+\frac{s_{1}+s_{2}}{2}\right) \rightarrow c-c z, \quad \text { as } \quad \varepsilon \rightarrow 0, \\
\varepsilon \frac{s_{2}-s_{1}}{2} \rightarrow c, \quad \text { as } \quad \varepsilon \rightarrow 0, \\
\frac{s_{1}+s_{2}}{s_{2}-s_{1}} \rightarrow 1, \quad \text { as } \quad \varepsilon \rightarrow 0, \\
b \rightarrow 0, \quad \text { as } \quad \varepsilon \rightarrow 0 .
\end{gathered}
$$

Define

$$
\bar{F}_{1}(z) \triangleq F(c-c z ; \sigma, 0) .
$$


Then we have

$$
\begin{aligned}
A_{11} \quad & \rightarrow \int_{0}^{1}\left(\frac{c}{\sqrt{\bar{F}_{1}(z)}}-\frac{1}{\sqrt{\frac{P_{v}\left(w_{0}\right)}{2}}} \frac{1}{1-z}\right) d z \\
& =c \int_{0}^{1}\left(\frac{1}{(1-z) \sqrt{\frac{\bar{F}_{1}(z)}{(1-z)^{2}}}}-\frac{1}{c \sqrt{\frac{P_{v}\left(w_{0}\right)}{2}}} \frac{1}{1-z}\right) d z \\
& :=c \int_{0}^{1} G(z) d z, \quad \text { as } \quad \varepsilon \rightarrow 0 .
\end{aligned}
$$

Note that $\bar{F}_{1}(1)=0, \bar{F}_{1}^{\prime}(1)=0$ and $\bar{F}_{1}^{\prime \prime}(1) \neq 0$. Hence, by Taylor's fomula, we have

$$
g(z) \triangleq \frac{\bar{F}_{1}(z)}{(1-z)^{2}} \rightarrow \frac{\bar{F}_{1}^{\prime \prime}(1)}{2}=\frac{P_{v}\left(w_{0}\right)}{2} c^{2}, \quad \text { as } \quad z \rightarrow 1,
$$

and

$$
g^{\prime}(z)=\frac{\bar{F}_{1}^{\prime \prime \prime}(1)}{6}+O((z-1)) \rightarrow \frac{\bar{F}_{1}^{\prime \prime \prime}(1)}{6}=\frac{-P_{v v}\left(w_{0}\right) c^{3}}{6}, \quad \text { as } \quad z \rightarrow 1 .
$$

Then we deduce that

$$
\begin{aligned}
\lim _{z \rightarrow 1} G(z) & =\lim _{z \rightarrow 1} \frac{1}{1-z}\left(\frac{1}{\sqrt{g(z)}}-\frac{1}{c \sqrt{\frac{P_{v}\left(w_{0}\right)}{2}}}\right) \\
& =\lim _{z \rightarrow 1} \frac{1}{2} g^{-\frac{3}{2}} g^{\prime}=-\frac{\sqrt{2}}{6} P_{v}\left(w_{0}\right)^{-\frac{3}{2}} P_{v v}\left(w_{0}\right) .
\end{aligned}
$$

Thus, $G(z)$ is a continuous function on $[0,1]$. So $A_{11}=O(1)$ as $\varepsilon \rightarrow 1$.

Similarly, we can obtain that

$$
A_{2} \rightarrow \int_{0}^{1} \frac{c}{\sqrt{\bar{F}_{2}(z)}} d z, \quad \text { as } \quad \varepsilon \rightarrow 0,
$$

where $\bar{F}_{2}(z) \triangleq F(c+c z ; \sigma, 0)$. Note that $\bar{F}_{2}(1)=F(\tilde{v} ; \sigma, 0)=0$. We have

$$
\lim _{z \rightarrow 1} \frac{\bar{F}_{2}(z)}{1-z}=\bar{F}_{2}^{\prime}(1)=c(P(\tilde{v})-\sigma) \neq 0 .
$$

Since the integral $\int_{0}^{1} \frac{d z}{\sqrt{1-z}}$ is convergent, we get $A_{2}=O(1)$ as $\varepsilon \rightarrow 1$.

In an analogous way, we obtain the following proposition.

Proposition 3.3. Let $\sigma \in\left(\frac{1}{2}, 1\right)$ be fixed. Then it follows that as $b \downarrow b_{2}(\sigma)$,

$$
\begin{aligned}
& I_{0}(\sigma, b) \rightarrow \frac{1}{2} \sqrt{\frac{k(1-\sigma)}{L}} \frac{1}{\sqrt{P_{v}\left(w_{2} ; \sigma\right)}} \log \frac{1}{b-b_{2}(\sigma)}+O(1), \\
& I_{1}(\sigma, b) \rightarrow \frac{1}{2} \sqrt{\frac{k(1-\sigma)}{L}} \frac{\log \frac{1}{b-b_{2}(\sigma)}}{\sqrt{P_{v}\left(w_{2} ; \sigma\right)}}\left[2 w_{2}^{3}-3 w_{2}^{4}-\frac{6 \sigma w_{2}^{2}\left(1-w_{2}\right)^{2}}{1-\sigma}+\frac{a \sigma}{1-\sigma}\right]+O(1), \\
& R(\sigma, b) \rightarrow \frac{a \sigma}{1-\sigma}-1 .
\end{aligned}
$$

In addition, for the behavior of $I_{0}(\sigma, b)$ and $R(\sigma, b)$ as we approach the Maxwell point $A=\left(\frac{1}{2}, 0\right)$ (cf. 23] ) along the line $\sigma=\frac{1}{2}$, we obtain the following result in a similar way. 
Proposition 3.4. Let $\sigma=\frac{1}{2}$. Then it follows that as $b \downarrow 0$,

$$
\begin{aligned}
& I_{0}(\sigma, b) \rightarrow \frac{1}{2} \sqrt{\frac{k(1-\sigma)}{L}}\left(\frac{1}{\sqrt{P_{v}\left(w_{0} ; \sigma\right)}}+\frac{1}{\sqrt{P_{v}\left(w_{2} ; \sigma\right)}}\right) \log \frac{1}{b}+O(1), \\
& I_{1}(\sigma, b) \rightarrow \frac{1}{2} \sqrt{\frac{k(1-\sigma)}{L}}\left(\frac{\frac{a \sigma}{1-\sigma}}{\sqrt{P_{v}\left(w_{0} ; \sigma\right)}}+\frac{\frac{a \sigma}{1-\sigma}-1}{\sqrt{P_{v}\left(w_{2} ; \sigma\right)}}\right) \log \frac{1}{b}+O(1), \\
& R(\sigma, b) \rightarrow \frac{\left(\frac{a \sigma}{1-\sigma}-1\right) \sqrt{P_{v}\left(w_{0} ; \sigma\right)}+\frac{a \sigma}{1-\sigma} \sqrt{P_{v}\left(w_{2} ; \sigma\right)}}{\sqrt{P_{v}\left(w_{0} ; \sigma\right)}+\sqrt{P_{v}\left(w_{2} ; \sigma\right)}} .
\end{aligned}
$$

4. Monotonicity of $I_{0}(\sigma, b)$. In this section, we study the monotonicity of $I_{0}$ with respect to $b$.

First, we recall some results on the general two-points boundary value problems given in [18] (see also [19, 23]). Consider the following problem:

$$
v_{x x}+f(v)=0, \quad v_{x}(0)=v_{x}(r)=0,
$$

where $f$ satisfies

$$
\begin{aligned}
& f(\eta)=0, \quad f^{\prime}(\eta)>0, \\
& f(v)=(v-\eta) f^{+}(v) \quad \text { with } \quad a^{-}<\eta<a^{+},
\end{aligned}
$$

and $f^{+}:\left(a^{-}, a^{+}\right) \rightarrow \mathbb{R}^{+}$is locally Lipschitz continuous. Thus,

$$
v_{x x}+f(v)=0, \quad v_{x}(0)=0, \quad v(0)=a,
$$

has a unique solution $x \mapsto V(x, a)$ for any $a \in\left(a^{-}, a^{+}\right)$. If $V(\cdot, a) \not \equiv$ Const., we can define the time map $\mathcal{T}$ of (4.3) by

$$
\begin{gathered}
D(\mathcal{T}):=\left\{a \in\left(a^{-}, a^{+}\right) \mid V(\cdot, a) \not \equiv \text { Const. } V^{\prime}(x, a)=0 \text { for some } x>0\right\} \\
\mathcal{T}(a):=\min \left\{x>0 \mid V^{\prime}(x, a)=0 \text { for } a \in D(\mathcal{T})\right\} .
\end{gathered}
$$

Then, we have the following lemma (see [18, Theorem 2.1.3]):

Lemma 4.1. Let (4.2) holds for $f$. Define $H$ to be the integral of $f$ with $H(\eta)=0$. Let $(\underline{a}, \bar{a})$ be the maximal subinterval of $\left(a^{-}, a^{+}\right)$such that $H(\underline{a})=H(\bar{a})$. Then, if $f$ is an A-B function on $\left(a^{-}, a^{+}\right)$, we have

$$
(a-\eta) \mathcal{T}^{\prime}(a)>0, \quad \text { for } \quad a \in(\underline{a}, \bar{a}) \backslash\{\eta\} .
$$

Next, in order to prove that $I_{0}$ is monotone decreasing in $b$, we examine the conditions of Lemma 4.1

Lemma 4.2. Let $f(v)=\frac{12 L}{k(1-\sigma)} v(1-v)(v-\sigma)$. Then for any fixed $\sigma \in(0,1), f$ is an A-B function on $(0,1)$, i.e.,

(1) $f^{\prime}$ has only simple zeros on $(0,1)$;

(2) $f^{\prime} f^{\prime \prime \prime}-\frac{5}{3}\left(f^{\prime \prime}\right)^{2}<0$ on any subinterval of $(0,1)$ on which $f^{\prime}$ is positive;

(3) $f f^{\prime \prime}-3\left(f^{\prime}\right)^{2} \leq 0$ on any subinterval of $(0,1)$ on which $f^{\prime}$ is negative. 
Proof. First, we calculate that

$$
\begin{gathered}
f^{\prime}(v)=-\frac{12 L}{k(1-\sigma)}\left(3 v^{2}-2(1+\sigma) v+\sigma\right), \\
f^{\prime \prime}(v)=-\frac{12 L}{k(1-\sigma)}(6 v-2(1+\sigma)),
\end{gathered}
$$

and

$$
f^{\prime \prime \prime}(v)=-\frac{72 L}{k(1-\sigma)} .
$$

For (11), we note that the discriminant of $f^{\prime}$ is positive on $(0,1)$. So condition (11) is satisfied.

For (2), we have that

$$
\begin{aligned}
f^{\prime} f^{\prime \prime \prime}-\frac{1}{2}\left(f^{\prime \prime}\right)^{2} & =\frac{144 L^{2}}{k^{2}(1-\sigma)^{2}}\left[6\left(3 v^{2}-2(1+\sigma) v+\sigma\right)-\frac{1}{2}(6 v-2(1+\sigma))^{2}\right] \\
& =-\frac{288 L^{2}}{k^{2}(1-\sigma)^{2}}\left(\sigma^{2}-\sigma+1\right)<0, \quad \text { when } \quad \sigma \in(0,1) .
\end{aligned}
$$

Then (2) follows easily.

For (3), we calculate that

$$
f f^{\prime \prime}-\frac{2}{3}\left(f^{\prime}\right)^{2}=\frac{96 L^{2}}{k^{2}(1-\sigma)^{2}}\left[\left(3 \sigma-(1+\sigma)^{2}\right) v^{2}+\sigma(1+\sigma) v-\sigma^{2}\right] .
$$

Noticing that by the Cauchy-Schwarz inequality,

$$
\sigma(1+\sigma) v \leq \sigma^{2}+\frac{(1+\sigma)^{2}}{4} v^{2}
$$

and

$$
3 \sigma-(1+\sigma)^{2}+\frac{(1+\sigma)^{2}}{4}=-\frac{3}{4}(1-\sigma)^{2} \leq 0,
$$

we deduce that $f f^{\prime \prime}-3\left(f^{\prime}\right)^{2} \leq 0$ and hence condition (3) follows and this completes the proof.

In conclusion, we deduce the following decreasing monotone property of $I_{0}$ with respect to $b$.

Proposition 4.1. It follows that

$$
\frac{\partial}{\partial b} I_{0}(\sigma, b)<0, \quad \text { for }(\sigma, b) \in \operatorname{Int} \Sigma .
$$

Proof. Note that for any $\sigma \in(0,1), f(v)=-\frac{12 L}{k(1-\sigma)} P(v ; \sigma)$. Then it follows from Lemma 4.2 that $f$ is an A-B function. Therefore, by Lemma 4.1, we have

$$
\frac{\partial}{\partial v_{1}} \tilde{I}_{0}\left(\sigma, v_{1}\right)<0
$$

where $\tilde{I}_{0}\left(\sigma, v_{1}\right)=I_{0}\left(\sigma, b\left(\sigma, v_{1}\right)\right)$. On the other hand, since $v_{1}(\sigma, b)$ is one of the zeros of $F(v ; \sigma, b)$, we deduce that

$$
\frac{\partial v_{1}}{\partial b}=\frac{1}{F_{v}\left(v_{1} ; \sigma, b\right)}>0 \quad \text { for }(\sigma, b) \in \operatorname{Int} \Sigma .
$$


Consequently, we have

$$
\frac{\partial}{\partial b} I_{0}(\sigma, b)=\frac{\partial}{\partial v_{1}} \tilde{I}_{0}\left(\sigma, v_{1}\right) \frac{\partial v_{1}}{\partial b}<0 \quad \text { for }(\sigma, b) \in \operatorname{Int} \Sigma .
$$

5. The level sets of $I_{0}(\sigma, b)$ and $R(\sigma, b)$. In this section, we examine the level sets

$$
\mathscr{C}_{\lambda}=\left\{(\sigma, b): I_{0}(\sigma, b)=\lambda\right\}
$$

and

$$
\mathscr{D}_{\mu}=\{(\sigma, b): R(\sigma, b)=\mu\}
$$

in $\Sigma$. Our aim is to count the number of the intersection points of the two level sets. To this end, we first study the number of their intersection points with the boundary of $\Sigma$.

Proposition 5.1. We have the following results for the level sets $\mathscr{C}_{\lambda}$ :

(i) $I_{0}>\underline{\lambda}:=\pi \sqrt{\frac{k}{12 L}}$ along $\Gamma_{1}$.

(ii) $\underline{\lambda}<\lambda<\infty \Leftrightarrow \mathscr{C}_{\lambda}$ and $\Gamma_{1}$ have exactly one intersection point.

(iii) We have $I_{0}>\underline{\lambda}$ in $\operatorname{Int} \Sigma$ and $\lambda \leq \underline{\lambda} \Leftrightarrow \mathscr{C}_{\lambda}$ and $\Gamma_{1}$ have no intersection point.

(iv) Denote $\mathscr{C}_{\infty}=\lim _{\lambda \rightarrow+\infty} \mathscr{C}_{\lambda}$. Then we have $\mathscr{C}_{\infty}=\overline{\Gamma_{2} \cup \Gamma_{3}} \backslash\{B\}$, where $B=(1,1)$.

Proof. These results follow directly from Proposition 3.1 Proposition 3.2 and Proposition 3.3

Recall that when $m / L \leq-1$, problem (1.11)-(1.12) has no solutions. Thus, we only have to consider the level set $\mathscr{D}_{\mu}$ for $\mu>-1$.

To begin with, we consider the following function:

$$
g(\sigma) \triangleq 3 \sigma^{4}-4 \sigma^{3}+\frac{a \sigma}{1-\sigma}, \quad 0<\sigma<1 .
$$

By a straightfoward calculation, we find that

$$
g^{\prime}(\sigma)=\frac{a-12 \sigma^{2}(1-\sigma)^{3}}{(1-\sigma)^{2}}
$$

and

$$
g^{\prime \prime}(\sigma)=36 \sigma^{2}-24 \sigma+2 a(1-\sigma)^{-3} .
$$

When $0<\sigma<1,12 \sigma^{2}(1-\sigma)^{3}$ attains its maximum at $\frac{2}{5}$ with the value $a_{0}=12 \times\left(\frac{2}{5}\right)^{2} \times$ $\left(\frac{3}{5}\right)^{3}$. Hence, $g(\sigma)$ is nondecreasing when $a \geq a_{0}$.

If $a<a_{0}, g(\sigma)$ has two critical points denoted by $\sigma_{1}$ and $\sigma_{2}$ satisfying $0<\sigma_{1}<\sigma_{2}<1$, $g^{\prime}(\sigma)>0$ when $\sigma \in\left[0, \sigma_{1}\right) \cup\left(\sigma_{2}, 1\right)$ and $g^{\prime}(\sigma)<0$ when $\sigma \in\left(\sigma_{1}, \sigma_{2}\right)$

It follows from $g^{\prime}\left(\sigma_{2}\right)=0$ that

$$
g\left(\sigma_{2}\right)=\frac{a \sigma_{2}}{12\left(1-\sigma_{2}\right)^{3}}\left(12 \sigma_{2}^{2}-21 \sigma_{2}+8\right) .
$$

Denote $\sigma^{*}=\frac{21-\sqrt{57}}{24}$. Then, if $\sigma_{2} \in\left(\frac{2}{5}, \sigma^{*}\right]$, we have $g\left(\sigma_{2}\right) \geq 0$ and if $\sigma_{2} \in\left(\sigma^{*}, 1\right)$, we have $g\left(\sigma_{2}\right)<0$. Correspondingly, denoting $a_{1}=12 \sigma^{* 2}\left(1-\sigma^{*}\right)^{3}$, we find that if $a_{1} \leq a<a_{0}$, then $g\left(\sigma_{2}\right) \geq 0$ and if $a<a_{1}$, it follows that $g\left(\sigma_{2}\right)<0$.

A sketch of $g(\sigma)$ with different $a$ is shown as in Figure 3. 


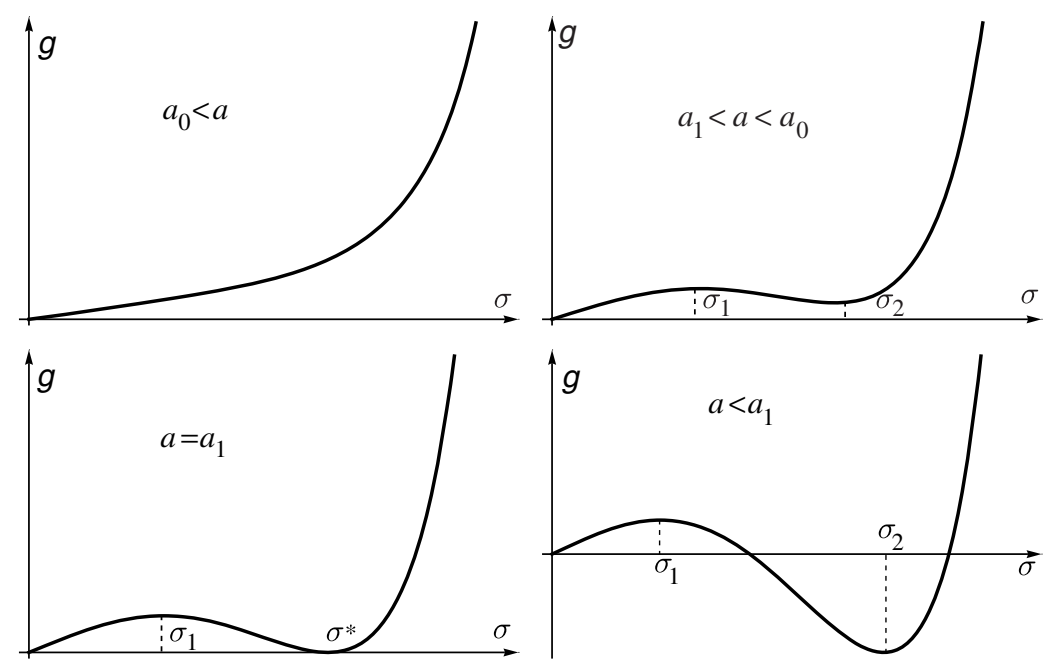

FIG. 3. A sketch of $g(\sigma)$.

Proposition 5.2. We have the following results for the level set $\mathscr{D}_{\mu}$ :

(i) If $a \geq 1$, we have four subcases.

When $\mu \in(-1,0], \mathscr{D}_{\mu}$ and $\Gamma_{0} \cup \Gamma_{1} \cup \Gamma_{2} \backslash\{A\}$ have no intersection point.

When $\mu \in(0, a-1]$ and $a>1, \mathscr{D}_{\mu}$ has one intersection point with $\Gamma_{1}$ and $\Gamma_{0} \backslash\{A\}$ respectively, while $\mathscr{D}_{\mu}$ and $\Gamma_{2} \backslash\{A\}$ have no intersection point.

When $\mu \in(a-1, a), \mathscr{D}_{\mu}$ has one intersection point with $\Gamma_{1}, \Gamma_{0} \backslash\{A\}$ and $\Gamma_{2} \backslash\{A\}$ respectively.

When $\mu \in[a,+\infty), \mathscr{D}_{\mu}$ has one intersection point with $\Gamma_{1}$ and $\Gamma_{2} \backslash\{A\}$ respectively, while $\mathscr{D}_{\mu}$ and $\Gamma_{0} \backslash\{A\}$ have no intersection point.

(ii) If $a_{0} \leq a<1$, we have four subcases.

When $\mu \in(-1, a-1], \mathscr{D}_{\mu}$ and $\Gamma_{0} \cup \Gamma_{1} \cup \Gamma_{2} \backslash\{A\}$ have no intersection point.

When $\mu \in(a-1,0], \mathscr{D}_{\mu}$ and $\Gamma_{2} \backslash\{A\}$ have one intersection point, while $\mathscr{D}_{\mu}$ and $\Gamma_{0} \cup \Gamma_{1} \backslash\{A\}$ have no intersection point.

When $\mu \in(0, a), \mathscr{D}_{\mu}$ has one intersection point with $\Gamma_{1}, \Gamma_{0} \backslash\{A\}, \Gamma_{2} \backslash\{A\}$, respectively.

When $\mu \in[a,+\infty), \mathscr{D}_{\mu}$ has one intersection point with $\Gamma_{1}$ and $\Gamma_{2} \backslash\{A\}$ respectively, while $\mathscr{D}_{\mu}$ and $\Gamma_{0} \backslash\{A\}$ have no intersection point.

(iii) If $a_{1}<a<a_{0}$, we have four subcases.

When $\mu \in(-1, a-1], \mathscr{D}_{\mu}$ and $\Gamma_{0} \cup \Gamma_{1} \cup \Gamma_{2} \backslash\{A\}$ have no intersection point.

When $\mu \in(a-1,0], \mathscr{D}_{\mu}$ and $\Gamma_{2} \backslash\{A\}$ have one intersection point, while $\mathscr{D}_{\mu}$ and $\Gamma_{0} \cup \Gamma_{1} \backslash\{A\}$ have no intersection point.

When $\mu \in(0, a), \mathscr{D}_{\mu}$ has one intersection point with $\Gamma_{0} \backslash\{A\}$ and $\Gamma_{2} \backslash\{A\}$ respectively while $\mathscr{D}_{\mu}$ and $\Gamma_{1}$ have at least one intersection point.

When $\mu \in[a,+\infty), \mathscr{D}_{\mu}$ and $\Gamma_{2} \backslash\{A\}$ have one intersection point, $\mathscr{D}_{\mu}$ and $\Gamma_{1}$ have at least one intersection point, while $\mathscr{D}_{\mu}$ and $\Gamma_{0} \backslash\{A\}$ have no intersection point.

(iv) If $0<a \leq a_{1}$, we have the following subcases.

When $\mu \in(-1, a-1], \mathscr{D}_{\mu}$ and $\Gamma_{0} \cup \Gamma_{1} \cup \Gamma_{2} \backslash\{A\}$ have no intersection point. 
When $\mu \in\left(a-1, g\left(\sigma_{2}\right)\right), \mathscr{D}_{\mu}$ and $\Gamma_{0} \cup \Gamma_{1} \backslash\{A\}$ have no intersection point; $\mathscr{D}_{\mu}$ and $\Gamma_{2} \backslash\{A\}$ have one intersection point.

When $\mu=g\left(\sigma_{2}\right), \mathscr{D}_{\mu}$ and $\Gamma_{1}$ and $\Gamma_{2} \backslash\{A\}$ have one intersection point respectively, while $\mathscr{D}_{\mu}$ and $\Gamma \backslash\{A\}$ has no intersection point.

When $\mu \in\left(g\left(\sigma_{2}\right), 0\right], \mathscr{D}_{\mu}$ and $\Gamma_{1}$ have two intersection points, $\mathscr{D}_{\mu}$ and $\Gamma_{2} \backslash\{A\}$ have one intersection point, while $\mathscr{D}_{\mu}$ and $\Gamma_{0} \backslash\{A\}$ have no intersection point.

When $\mu \in\left(0, g\left(\sigma_{1}\right)\right), \mathscr{D}_{\mu}$ and $\Gamma_{1}$ have three intersection points while $\mathscr{D}_{\mu}$ and $\Gamma_{i} \backslash\{A\}$ $(i=0,2)$ have one intersection point.

When $\mu=g\left(\sigma_{1}\right), \mathscr{D}_{\mu}$ and $\Gamma_{1}$ have two intersection points while $\mathscr{D}_{\mu}$ and $\Gamma_{i} \backslash\{A\}$ $(i=0,2)$ have one intersection point.

When $\mu \in\left(g\left(\sigma_{1}\right), a\right), \mathscr{D}_{\mu}$ and $\Gamma_{1}, \Gamma_{i} \backslash\{A\}(i=0,2)$ have one intersection point.

When $\mu \in[a,+\infty), \mathscr{D}_{\mu}$ and $\Gamma_{1}, \Gamma_{2} \backslash\{A\}$ have one intersection point, while $\mathscr{D}_{\mu}$ and $\Gamma_{0} \backslash\{A\}$ have no intersection point.

Proof. First, we note that $A$ is a singularity of $R(\sigma, b)$ in $\Sigma$. Assertions (i)-(iii) follow easily from the property of $g(\sigma)$ and Propositions 3.1]3.4

For (iv), we point out that if $0<a<a_{1}$, it is not difficult to deduce from (5.6) and the property of function $y(s):=12 s^{2}(1-s)^{3}$ for $s \in(0,1)$ that $\sigma_{2}>\sigma^{*}>\frac{1}{2}>\frac{2}{5}>\sigma_{1}$. On the other hand, when $\sigma \in\left(\frac{1}{2}, 1\right)$, we infer that

$$
a-1<\frac{a \sigma}{1-\sigma}-1<g(\sigma)
$$

and when $\sigma \in\left(0, \frac{1}{2}\right)$,

$$
g(\sigma)<\frac{a \sigma}{1-\sigma}<a .
$$

Then the conclusion can be deduced by delicate discussions.

Before concluding this section, we demonstrate the analyticity of $I_{0}(\sigma, b)$ and $R(\sigma, b)$ in $\operatorname{Int} \Sigma$.

Proposition 5.3. $I_{0}(\sigma, b)$ and $R(\sigma, b)$ are analytic on the parameters $(\sigma, b)$ in $\operatorname{Int} \Sigma$.

Proof. We first prove that $v_{i}(\sigma, b)(i=1,2)$ are analytic on $(\sigma, b)$ in Int $\Sigma$. Recall that

$$
F\left(v_{i} ; \sigma, b\right)=\Phi\left(v_{i} ; \sigma\right)-b=3 v_{i}^{4}-4(1+\sigma) v_{i}^{3}+6 \sigma v_{i}^{2}-b=0 .
$$

Obviously, $F(v ; \sigma, b)$ is analytic on $v, \sigma, b$ and $F_{v}\left(v_{i}\right) \neq 0$ for $(\sigma, b) \in \operatorname{Int} \Sigma$. By the implicit function theorem of analytic functions, we obtain that $v_{i}(\sigma, b)(i=1,2)$ are analytic on $(\sigma, b)$ in Int $\Sigma$. Thus $Q\left(x ; v_{i}, w_{1}\right)$ defined in (3.3) is also analytic in $\sigma$ and $b$. Recalling that

$$
\sqrt{\frac{2 L}{k(1-\sigma)}} I_{0}(\sigma, b)=\sum_{i=1}^{2} \int_{0}^{1} \frac{d x}{\sqrt{(1-x) Q\left(x ; v_{i}, w_{1}\right)}},
$$

we obtain that $I_{0}(\sigma, b)$ is analytic on the parameters $(\sigma, b)$ in Int $\Sigma$. The analyticity of $I_{1}(\sigma, b)$ can be proved in the same manner. Thus, by (2.9) and the fact that $I_{0}>\underline{\lambda}>0$ in $\operatorname{Int} \Sigma$, the analyticity of $R(\sigma, b)$ follows.

Corollary 5.1. (a) For any $\lambda>\underline{\lambda}$, the level set $\mathscr{C}_{\lambda}$ consists of a single connected curve joining the points $\left(\frac{\pi^{2} k}{12 \lambda^{2} L},\left(\frac{\pi^{2} k}{12 \lambda^{2} L}\right)^{3}\left(2-\frac{\pi^{2} k}{12 \lambda^{2} L}\right)\right) \in \Gamma_{1}$ and $B$. 
(b) The set $\mathscr{D}_{\mu}$ consists of at most a countably infinite number of curves which are either closed or else have end-points on the boundary of $\Sigma$ (under the constraints of Proposition 5.2). Moreover, it follows that

$$
\sup _{(\sigma, b) \in \mathscr{D}_{\mu}} \sigma \leq \frac{1+\mu}{1+\mu+a}<1 .
$$

Proof. For (a), combining the continuity of $I_{0}(\sigma, b)$ in Int $\Sigma$ with the monotone decreasing property of $I_{0}$ with respect to $b$, and noticing that $I_{0}(\sigma, b)$ is decreasing with respect to $\sigma$ along $\Gamma_{1}$, we infer that for any $(\hat{\sigma}, \hat{b}) \in \operatorname{Int} \Sigma$ satisfying $I_{0}(\hat{\sigma}, \hat{b})=\lambda>\underline{\lambda}$, and $\forall \sigma \in(\hat{\sigma}, 1)$, there exists a unique point $(\sigma, b) \in \operatorname{Int} \Sigma$ such that $I_{0}(\sigma, b)=\lambda$. Thus, by Proposition 3.1, we obtain that for any $\lambda>\underline{\lambda}$, the set $\mathscr{C}_{\lambda}$ consists of a single connected curve joining the points $\left(\frac{\pi^{2} k}{12 \lambda^{2} L},\left(\frac{\pi^{2} k}{12 \lambda^{2} L}\right)^{3}\left(2-\frac{\pi^{2} k}{12 \lambda^{2} L}\right)\right) \in \Gamma_{1}$ and $B$. A sketch of the level sets $\mathscr{C}_{\lambda}$ for $\lambda>\underline{\lambda}$ is shown in Figure 4 .

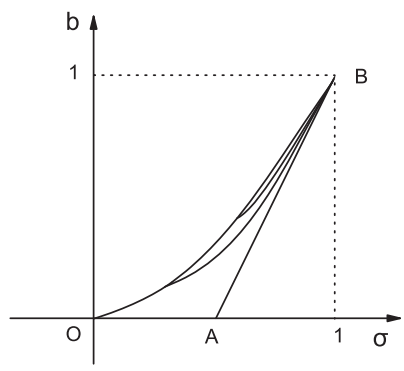

FIG. 4. A sketch of $\mathscr{C}_{\lambda}$ for $\lambda>\underline{\lambda}$.

For (b), we use the analyticity of $R(\sigma, b)$, (1.6) and (1.10).

6. Proofs of the main theorems. In this section, we prove our main Theorems based on results previously obtained.

Proof of Theorem 1.1. Firstly, (1) follows directly from Proposition 3.1 and Proposition 4.1

Now we prove (2) and (3) together.

In view of Corollary 5.1 and properties (P1)-(P3), we deduce that the total number of nontrivial solutions to problem (1.12) is $N=2 \sum N_{k}$, where

$$
N_{k}=\left\{\begin{array}{c}
\text { the total number of points }(\sigma, b) \in \Sigma \text { such that } \\
I_{0}(\sigma, b)=l / n \text { and } R(\sigma, b)=m / L, n=1,2, \ldots,[l / \underline{\lambda}]
\end{array}\right\}
$$

is the number of $\mathscr{C}_{l / n} \cap \mathscr{D}_{m / L}$ for any fixed $n \in\{1,2, \ldots,[l / \underline{\lambda}]\}$.

For any $\lambda$ and $\mu$, the level sets $\mathscr{C}_{\lambda}$ do not coincide with any curve component of $\mathscr{D}_{\mu}$ because the end-points of them do not coincide according to (5.11). Noticing that $\partial I_{0} / \partial b<0$, we get $b=b_{l / n}(\sigma)$ from $I_{0}(\sigma, b)=l / n$. Then substituting it into $R(\sigma, b)=$ $m / L$ leads to $R\left(\sigma, b_{l / n}(\sigma)\right)=m / L$. Therefore, by the analyticity of $R\left(\sigma, b_{l / n}(\sigma)\right)$ on $\sigma \in(0,1)$, we know that the set $\mathscr{C}_{l / n} \cap \mathscr{D}_{m / L}$ has no accumulation point in Int $\Sigma$. Thus, there exists an accumulation point of $\mathscr{C}_{l / n} \cap \mathscr{D}_{m / L}$ on $\partial \Sigma$ if and only if: 
$a, m / L$ are not in the cases $(2)(\mathrm{a})-(2)(\mathrm{c})$, and $m / L=3 \sigma_{0}^{4}-4 \sigma_{0}^{3}+\frac{a \sigma_{0}}{1-\sigma_{0}}$ for some $n \in$ $\{1,2, \ldots,[l / \underline{\lambda}]\}$, where $\sigma_{0}=\frac{\pi^{2} k}{12 L}\left(\frac{n}{l}\right)^{2}$. This completes the proof.

Proof of Theorem 1.2. From [5, Theorem 1.1], we know that for any $\left(f_{0}, \theta_{0}\right) \in H^{3} \times H^{1}$ satisfying $\left.f_{0 x}\right|_{x=0, l}=0$ and $\theta_{0}>0$ in $[0, l]$, the $\omega$-limit set $\omega\left(f_{0}, \theta_{0}\right)$ is a nonempty connected compact set and consists of stationary solutions. On the other hand, it was proven in [5, Theorem 3.1] that

$$
\|\theta-\bar{\theta}\|_{L^{\infty}} \rightarrow 0
$$

and by the Jensen inequality it follows that (cf. [5, Lemma 5.2])

$$
\bar{\theta}:=\frac{1}{l} \int_{0}^{l} \theta d x \geq C>0,
$$

where $C$ is a positive constant depending only on the initial data and the coefficients of the system. Thus, the $\omega$-limit set consists of regular solutions to (1.11)-(1.12). Since we have proved in Theorem 1.1 that there exists at most a countably infinite number of such stationary solutions, then the $\omega$-limit set is discrete and the results follow directly.

Acknowledgment. The authors would like to express their sincere thanks to Prof. Songmu Zheng for his helpful discussions. The second author, Y. Zhang, was supported by the Tianyuan Fund of China under the grant 11126235.

\section{REFERENCES}

[1] V. Berti, M. Fabrizio and C. Giorgi, Well-posedness for solid-liquid phase transition with a fourthorder nonlinearity, Physica D, 236 (2007), 13-21. MR2385021 (2008j:80007)

[2] G. Bonfanti, M. Frémond and F. Luterotti, Global solution to a nonlinear system for irreversible phase changes, Adv. Math. Sci. Appl., 10 (2000), 1-24. MR1769184 (2001g:35272)

[3] M. Frémond, Non-smooth Thermomechanics, Springer-Verlag, Berlin, 2002. MR1885252 (2003g:74004)

[4] M. Grinfeld and A. Novick-Cohen, Counting stationary solutions of the Cahn-Hilliard equation by transversality arguments, P. Roy. Soc. Edinburgh. A. 125 (1995) 351-370. MR1331565 (96c:58157)

[5] J. Jiang and B. Guo, Asymptotic behavior of solutions to a one-dimensional full model for phase transitions with microscopic movements, Discr. Cont. Dyn. Syst.-A, to appear.

[6] Ph. Laurençot, G. Schimperna and U. Stefanelli, Global existence of a strong solution to the onedimensional full model for irreversible phase transitions, J. Math. Anal. Appl., 271 (2002), 426-442. MR 1923644 (2003j:80003)

[7] S. Lojasiewicz, Une propriété topologique des sous-ensembles analytiques réels, Colloques internationaux du C.N.R.S.: Les équations aux dérivées partielles, Paris (1962), Editions du C.N.R.S., Paris, 1963, 87-89. MR0160856 (28:4066)

[8] S. Lojasiewicz, Ensembles semi-analytiques, Preprint, I.H.E.S., Bures-sur-Yvette, 1965.

[9] S. Lojasiewicz and M. A. Zurro, On the gradient inequality, Bull. Polish Acad. Sci. Math., 47(1999), 143-145. MR:1686676 (2001b:32010)

[10] F. Luterotti and U. Stefanelli, Existence result for the one-dimensional full model of phase transitions, Z. Anal. Anwendungen, 21 (2002), 335-350. MR 1915265 (2003e:80010)

[11] F. Luterotti and U. Stefanelli, Errata and addendum to: "Existence result for the one-dimensional full model of phase transitions", [Z. Anal. Anwendungen, 21 (2002), 335-350], Z. Anal. Anwendungen, 22 (2003), 239-240. MR.1962087

[12] H. Matano, Convergence of solutions of one-dimensional semilinear heat equations, J. Math. Kyoto Univ., 18 (1978), 221-227. MR501842 (80a:35016)

[13] A. Novick-Cohen and M. Grinfeld, Counting the stationary states of the Sivashinsky equation, Nonlinear Anal. 24 (6)(1995), 875-881. MR.1320694 (96a:35175)

[14] A. Novick-Cohen and L. A. Peletier, Steady states of the one-dimensional Cahn-Hilliard equation, P. Roy. Soc. Edinburgh. A. 123 (1993), 1071-1098. MR.1263907 (94m:35272) 
[15] A. Novick-Cohen and L. A. Peletier, The steady states of one-dimensional Sivashinsky equations, Quart. Appl. Math. 50 (4) (1992), 759-777. MR.1193665 (93m:35046)

[16] A. Novick-Cohen and S. Zheng, The Penrose-Fife-type equations: counting the one-dimensional stationary solutions, P. Roy. Soc. Edinburgh. A. 126 (3) (1996), 483-504. MR1396275 (97f:35218)

[17] E. Rocca and R. Rossi, Global existence of strong solutions to the one-dimensional full model for phase transition in thermoviscoelastic materials, Applications of Mathematics, 53 (2008), 485-520. MR2469589 (2009k:35241)

[18] R. Schaaf, Global Solution Branches of Two Point Boundary Value Problems, Springer Lecture Notes in Mathematics, Vol. 1458, Springer, Berlin, 1990. MR1090827 (92a:34003)

[19] R. Schaaf, Global behaviour of solution branches for some Neumann problems depending on one or several parameters, J. Reine Angew. Math. 346 (1984), 1-31. MR.727393 (85i:58035)

[20] W. Shen and S. Zheng, On the coupled Cahn-Hilliard equations, Commun. Part. Diff. Eq. 18 (1993) 701-727. MR 1214877 (94j:35179)

[21] L. Simon, Asymptotics for a class of non-linear evolution equations, with applications to geometric problems, Annals Mathematica, 118 (1983), 525-571. MR.727703 (85b:58121)

[22] Y. Zhang, Counting the stationary states and the convergence to equilibrium for the 1-D thin film equation, Nonlinear Anal., 71 (2009), 1425-1437. MR2524358 (2010i:35164)

[23] Y. Zhang, The steady states and convergence to equilibria for a 1-D chemotaxis model with volumefilling effect, Math. Meth. Appl. Sci., 33 (2010), 25-40. MR2591221

[24] S. Zheng, Asymptotic behavior of solution to the Cahn-Hilliard equation, Appl. Anal. 23 (1986) 165-184. MR870486 (88b:35036)

[25] S. Zheng, Nonlinear Parabolic Equations and Hyperbolic-Parabolic Coupled Systems, Pitman Series Monographs and Surveys in Pure and Appl. Math., Vol. 76, Longman, London, 1995. MR1375458 (97g:35078)

[26] S. Zheng, Nonlinear Evolution Equations, Chapman \& Hall/CRC, Boca Raton, Florida, 2004. MR2088362 (2006a:35001) 